\title{
Statistically Modelling the Relationships between Type D Personality and Social Support, Health Behaviors and Symptom Severity in Chronic Illness Groups
}

\author{
Sharon Horwood, Jeromy Anglim, \& Greg Tooley \\ Deakin University ${ }^{1}$
}

Citation Information: Horwood, S., Anglim, J., \& Tooley, G. (2016). Statistically Modelling the Relationships between Type D Personality and Social Support, Health Behaviors and Symptom Severity in Chronic Illness Groups. Psychology \& Health, 31, 10471063. http://dx.doi.org/10.1080/08870446.2016.1167209

\begin{abstract}
Objective: The study aimed to develop a predictive model of how Type D personality influences health behaviors, social support, and symptom severity and assess its generalizability to a range of chronic illnesses. Design: Participants were classified as either healthy $(n=182)$ or having a chronic illness $(n=207)$. Participants completed an online survey measuring Type D and a range of health-related variables. Chronic illness participants were classified as having either a functional somatic syndrome (i.e. chronic fatigue syndrome or fibromyalgia), where the underlying pathological processes were unclear, or illnesses such as type 2 diabetes, osteoarthritis, or rheumatoid arthritis, where the causes are well understood. Main Outcome Measures: Outcome measures were health behaviors, social support, and both physical and psychological symptoms. Results: The rate of Type D was higher in chronic illness participants (53\%) than in healthy controls (39\%). Negative affectivity (NA) and social inhibition (SI) both correlated with outcome measures, although NA was generally the stronger predictor. Using NA and SI as independent subscales led to superior prediction of health outcomes than using categorical or continuous representations. Conclusion: Findings suggest that the relationship between Type D and health outcomes may generalize across different chronic illnesses.

Keywords: Type D, personality, somatization, functional somatic syndrome, chronic illness
\end{abstract}

\section{Introduction}

Type D personality is defined as the presence of high levels of both negative affectivity and social inhibition. Negative affectivity represents the tendency to experience negative emotions, and social inhibition represents the tendency to withhold negative emotions for fear of rejection or judgment by others (Denollet, 2005). People with Type D personality are theorized to engage in more deleterious health-related behaviors and perceptions than those without Type D (Denollet \& Pedersen, 2008; Williams et al., 2008), due largely to Type D representing a general susceptibility to psychological distress. Type D has been associated with a variety of negative health outcomes in cardiovascular disease

${ }^{1}$ This article may not exactly replicate the authoritative document published in the journal. It is not the copy of record. The link provided by the above doi links to the copy of record.

Sharon Horwood, Jeromy Anglim, and Greg Tooley, School of Psychology, Deakin University. Correspondence concerning this article should be addressed to Sharon Horwood, School of Psychology, Deakin University, 221 Burwood Highway, Burwood, 3125 Victoria, Australia. Email: sharon.horwood@deakin.edu.au 
(Pedersen \& Schiffer, 2011) and chronic heart failure patients (Conraads et al., 2006), and more recently in conditions such as cancer (Mols, Denollet, Kaptein, Reemst, \& Thong, 2012) type 2 diabetes (Nefs et al., 2015), Parkinson's disease (Dubayova et al., 2013), ulcerative colitis (Sajadinejad, Molavi, Asgari, Kalantari, \& Adibi, 2012) and migraine (Chan $\&$ Consedine, 2014). Although most Type D research has focused on cardiovascular disease and chronic heart failure patients, the generality of the mechanisms thought to underpin the relationship between Type D and poor health outcomes suggest that Type D could influence a broader range of chronic conditions.

Despite extensive research on Type D personality, several gaps remain. First, although Type D research has focused on its role in particular diseases, we are not aware of research that has compared the relationship between Type D and health status in healthy controls with chronic illness groups, in order to examine whether Type D represents a generalized risk factor for negative health outcomes and symptom experiences. Second, a range of debates have emerged about how Type D should be represented and integrated into models of health outcomes. Specifically, these debates include (a) whether Type D is dimensional or dichotomous, (b) whether the two subscales of type D have interactive or only additive effects, (c) whether the two subscales are equally relevant to disease processes, and (d) whether the effect of Type D on general health outcomes differs between chronic illnesses (Ferguson et al., 2009; Horwood, Anglim, \& Tooley, 2015). Thus, the purpose of the present study was develop and assess the generalizability of a model of Type D on health outcomes in both healthy controls and several high-prevalence, high-impact chronic conditions. As part of building such a model we aimed to contribute to the ongoing debates about the representation of Type D.

\subsection{Type D Personality}

Type D is typically diagnosed by scoring above a threshold on both negative affectivity and social inhibition subscales of the DS14, the standard measure of Type D personality (Denollet, 2005). These subscales share some overlap with the Big 5 traits of neuroticism and introversion respectively (Horwood et al., 2015). Type D personality is present in healthy and clinical populations with some evidence suggesting greater prevalence in specific illnesses. Estimates of Type D prevalence in healthy populations have varied from 13\% (Mommersteeg, Kupper, \& Denollet, 2010) to 24\% (Pedersen \& Denollet, 2004; Zohar, Denollet, Lev Ari, \& Cloninger, 2011) to 38\% (Horwood, Chamravi, \& Tooley, 2014; Williams et al., 2008). In cardiovascular and cardiac samples the rate is around $21 \%$ to $31 \%$ (Mols \& Denollet, 2010a), however in non-cardiac clinical samples estimates include 19\% in cancer patients (Husson, Denollet, Oerlemans, \& Mols, 2013), 29\% in type 2 diabetes patients (Nefs et al., 2015), and 59\% for female patients with ulcerative colitis (Sajadinejad et al., 2012). This variation in prevalence may suggest that Type D personality is either a greater risk for, or consequence of, some illnesses than others.

\subsection{Type D Personality and Health Outcomes}

Type D personality is thought to influence health status via a number of interacting biopsychosocial mechanisms. Individuals with Type D personality experience a range of heightened negative emotions such as worry and fear, and possess a negative view of the world, others, and themselves (Denollet, 2005). Additionally, their increased social inhibition means that they are less likely to outwardly express their distress (Denollet et al., 2006) and more likely to engage in maladaptive coping strategies, such as resignation and withdrawal (Martin et al., 2011; Polman, Borkoles, \& Nicholls, 2010). Type D individuals tend to report a greater range and number of symptoms, and perceive their condition as being more serious and prolonged than non-Type D patients (Jellesma, 2008). Nevertheless, they are less likely 
to engage in constructive health behaviors to maintain or improve their health status (Pelle, Schiffer, Smith, Widdershoven, \& Denollet, 2010; Williams et al., 2008).

Recent studies have reported evidence of HPA axis dysregulation in Type D patient groups, indicating a physiological dimension to the way in which the Type D profile may negatively affect health outcomes (Molloy, Perkins-Porras, Strike, \& Steptoe, 2008; Whitehead, Perkins-Porras, Strike, Magid, \& Steptoe, 2007). After adjusting for depression, Type D personality independently predicted increased cortisol levels in healthy individuals (Habra, 2003) and both increased cortisol (Whitehead et al., 2007) and oxidative stress (Kupper, Gidron, Winter, \& Denollet, 2009) in cardiac patients. As such, it is likely that maladaptive psychological and behavioral responses to stress that are linked to the Type D profile are also associated with chronic physiological changes that are deleterious to the health of the individual by increasing susceptibility to disease and aging (Habra, 2003; Rosmond \& Björntorp, 2000) .

While Type D research has focused mainly on cardiovascular diseases, the possible mechanisms of action described above could have influential roles in other high-prevalence and high-impact chronic illnesses. Researchers examining the role of Type D in conditions such as type 2 diabetes (e.g. Nefs et al., 2015), metabolic syndrome (e.g. Mommersteeg et al., 2010), and cancer (Mols et al., 2012) have found that Type D is associated with poorer mental and physical health status, and prolonged illness duration. Following a systematic review of Type D in the general population, Mols and Denollet (2010b) reported that Type D was associated with increased physical and mental health problems and disease promoting mechanisms in non-clinical, and even healthy populations. Michal et al. (2011) reported that Type D individuals were at severely increased risk for mental distress, major psychosocial stressors, and increased health care utilization. In a sample of over 3000 cancer survivors, Mols et al. (2012) found that Type D patients reported significantly higher levels of general somatic symptoms, sleep disturbance, pain, and fatigue. Similarly, in a recent population survey of more than 5000 Swedish adolescents, Type D was associated with higher levels of self-reported psychosomatic symptoms, musculoskeletal pain, and sleep disturbance (Condén, Leppert, Ekselius, \& Åslund, 2013; Condén, Rosenblad, Ekselius, \& Aslund, 2014). Other somatic research has found that negative affectivity and social inhibition are also each independently associated with increased somatization and unexplained symptoms (Watson \& Pennebaker, 1989; Wongpakaran \& Wongpakaran, 2014). Because, even in healthy populations, Type D personality is associated with somatic complaints and exaggerated symptom reporting, we hypothesize that functional somatic syndromes, conditions that are characterized primarily by general somatic complaints of unclear etiology, such as chronic fatigue syndrome or fibromyalgia, may be more susceptible to the effects of Type D personality than illnesses of known etiology such as type 2 diabetes or arthritis.

\subsection{Representations of Type D in Models of Health Outcomes}

Type D has been conceptualized traditionally as a categorical construct resulting from the combined effects of high negative affectivity and high social inhibition (Denollet, Pedersen, Vrints, \& Conraads, 2013; Denollet et al., 1996). This implies several questionable assumptions about the effect of Type D on health related outcomes. First, it assumes that negative affectivity and social inhibition have an interactive effect that is greater than the sum of the two main effects. Second, it implies that the main effects of negative affectivity and social inhibition are of similar importance in predicting health outcomes. Third, it suggests that there is a point of sharp discontinuity in the combined effect of social inhibition and negative affectivity on health outcomes, as opposed to a more linear effect that one would expect from a continuous variable.

Assessing personality in a dichotomous fashion almost always discards meaningful variance and has the potential to misclassify people who fall close to either side of the split 
(Haslam, Holland, \& Kuppens, 2012; Vollrath \& Torgersen, 2002). The reduced prediction of dichotomous variables holds whether using a single dichotomous Type $\mathrm{D}$ variable or creating multiple groups based on combinations of dichotomous NA and SI (e.g., Denollet et al., 2013). Recently several researchers have suggested that conceptualizing Type D as a dimensional construct is more consistent with personality trait theory, and should lead to greater predictive validity of health outcomes (e.g. see Bergvik, Sørlie, Wynn, \& Sexton, 2010; Ferguson et al., 2009; Horwood et al., 2015; Kelly-Hughes, Wetherell, \& Smith, 2014; Romppel, Herrmann-Lingen, Vesper, \& Grande, 2012). Researchers have examined measures of Type D both as the sum (Horwood et al., 2015) and the product (Stevenson \& Williams, 2014) of the two Type D subscales. However, there is limited research systematically comparing different representations of Type $\mathrm{D}$ in terms of predictive validity for health outcomes. This is a necessary step for conceptualization and practical utility of Type D in health research.

\subsection{The Current Study}

The present study aimed to develop models of how Type D personality influences health behaviors, social support, and symptom severity in chronic illness. We aimed to assess both the generalizability of models of Type $\mathrm{D}$ on health outcomes and refine understanding of how Type D personality should be represented in predictive models. To achieve these aims, we obtained a sample of healthy controls as well as individuals with a chronic illness that were categorized as having either a functional somatic syndrome (i.e., chronic fatigue syndrome or fibromyalgia) or an illness of clear etiological origins (i.e., type 2 diabetes, rheumatoid arthritis, or osteoarthritis). We examined predictive models of Type D on perceived social support, health behaviors, and physical and psychological symptoms. Specifically, we compared models using different representations of Type D and examined whether the effect of Type D varied between healthy and chronic illness groups and between functional somatic syndromes and illnesses of known etiology.

Consistent with previous prevalence research, we expected that the rate of Type D personality would be higher in chronic illness participants compared to healthy controls. As one of the characteristics of Type D personality is the tendency to report more somatic symptoms (Mols \& Denollet, 2010b), we also predicted that the rate of Type D would be higher in functional somatic syndrome's compared to illnesses of known etiology. Based on recent findings by Kelly-Hughes, Wetherell and Smith (2014) and Stevenson and Williams (2014) we also predicted that negative affectivity and social inhibition would be superior in predicting health behaviors, social support, and symptom severity over categorical or continuous representations of Type D. Finally, we also predicted that Type D would differentially predict health behaviors, social support, and symptom severity between healthy controls and chronic illness sufferers, and between functional somatic syndromes and illnesses of known etiology.

\section{Method}

\subsection{Participants and Procedure}

Participants were recruited via a number of illness support agencies (Diabetes Australia, CFS/ME Australia, FMS Support Australia) and social media sites (predominantly Facebook and Twitter). Participants completed an online survey composed of demographic questions, the DS14, the General Preventative Health Behaviors Checklist, the Social Network Support Scale and finally the Rotterdam Symptom Checklist. Participants were asked to respond yes or no to the follow statement regarding their health status: "Do you have a chronic illness that has been diagnosed by your GP or health care specialist? A chronic illness is defined as an illness that lasts at least six months in duration". Participants could 
select any of five chronic conditions; chronic fatigue syndrome, fibromyalgia, type 2 diabetes, rheumatoid arthritis, and osteoarthritis, or enter free text for any condition that differed from, or was comorbid with, any of the five under investigation. Ethics approval for this study was granted by our university Human Research Ethics Committee.

The final sample consisted of 389 participants after excluding participants with comorbid conditions $(n=60)$. This exclusion criteria was implemented to facilitate clear groupings, as well as exclude participants with conditions such as depression that may falsely inflate the negative affectivity or social inhibition scores of the DS14. Participants included 208 chronic illness participants and 181 healthy controls. Chronic illness participants were classified as either (a) functional somatic syndrome $(n=100)$ if they had a diagnosis of chronic fatigue syndrome or fibromyalgia, or (b) illnesses of known etiology $(n=107)$ if they had a diagnosis of type 2 diabetes, rheumatoid arthritis, or osteoarthritis. The sample was aged between 18 and 77 years $(M=37.8, S D=15.0)$ and $80.5 \%$ female. Most participants were born in Australia (76.6\%) and 3.3\% identified as Indigenous Australians. There was no significant differences between the healthy and chronic illness groups on age, gender, or ethnicity, however illnesses of known etiology participants were slightly older than those with a functional somatic syndrome, possibly due to the age-related degeneration associated with osteoarthritis.

\subsection{Materials}

Type D Personality Scale - DS14. The DS14 is a 14-item scale designed to measure the presence of Type D personality (Denollet, 2005). It uses a five-point response scale $(0=$ false, $1=$ rather false, 2 = neutral, 3 = rather true, $4=$ true) and consists of two 7 -item subscales: negative affectivity (NA) and social inhibition (SI). Using standard scoring, the DS14 provides subscale scores and a dichotomous diagnosis of Type D. Subscales are scored as the sum of item scores after item reversal. A diagnosis of Type D personality requires a score of 10 or more for both NA and SI subscales (Denollet, 2005). In a study of over 3000 participants, Denollet (2005) found that both subscales were internally consistent (Cronbach's $\alpha$ of .88 and .86 respectively), stable over a 3-month period (test-retest $r=.72$ and .82 ). The present study found Cronbach's $\alpha$ of .89 for NA and .87 for SI. Given arguments for conceptualizing Type D as a continuous construct (Ferguson et al., 2009; Kelly-Hughes et al., 2014) we also computed a continuous Type D (product) variable (i.e., product of NA and SI) and a continuous Type D (sum) variable (i.e., the sum of NA and SI).

Rotterdam Symptom Checklist. The Rotterdam Symptom Checklist (RSCL) (De Haes, 1990) is a 35-item scale used to measure the number of symptoms a person has experienced in the previous week. The measure uses a 4-item response scale where $1=$ not at all, $2=$ a little, $3=$ moderately, $4=$ very much. The measure is comprised of two subscales, physical symptoms (e.g. chest pain, headaches) and psychological symptoms (e.g. depressed mood, anxious feelings). Scores for symptom severity are represented as the sum of items. Reliability and convergent validity for the RSCL is moderate to strong, 0.8 and 0.6 respectively (Pelayo-Alvarez, Perez-Hoyos, \& Agra-Varela, 2013). The present study found Cronbach's $\alpha$ of .92 for psychological symptoms and .93 for physical symptoms.

General Preventative Health Behavior Checklist. The General Preventive Health Behaviors (GPHB) checklist measures the degree to which an individual engages in preventative health-related behaviors and provides a global index of health behavior. The GPHB checklist asks participants to indicate to what degree they perform 29 different healthrelated behaviors, answering on a 3 -point scale $(0=$ do not do it, $1=$ sometimes do it, $2=$ yes always, or almost always do it). Examples of scale items include 'Do not smoke', 'Limit alcohol intake', and 'Get a regular medical check-up'. The present study obtained a Cronbach's $\alpha$ statistic of .82. 
Social Network Support Scale. The Quality of Social Network and Social Support (SNSS) (Dalgard, Bjørk, \& Tambs, 1995) questionnaire measures the overall quality of perceived social support. We used the shortened 9-item version, as used in Williams et al. (2008), that measures social support with friends and family but not neighbors. Response scales varied across items but were either on a 0 to 3 or 0 to 2 scale. The measure showed good reliability in the current study (Cronbach's $\alpha=.78$ ).

\subsection{Data Analytic Approach}

We first compared the three groups on Type D prevalence (using categorical Type D), continuous Type D subscales, and health outcomes. We then examined the bivariate correlations between Type D subscales and outcome variables both for healthy and chronic illness participants. In order to contribute to the debate on how best to represent Type D, we compared the predictive validity (i.e., adjusted r-squared) of different representations of Type $\mathrm{D}$ predicting each health outcome. This involved comparing a range of categorical and continuous representations of Type D with and without interactions. The best predicting representation involved continuous SI and NA. There was also preliminary evidence for an SI by NA interaction. Thus, this representation was used in the subsequent regression models of Type D and chronic illness predicting health outcomes.

\section{Results}

\subsection{Group Differences and Correlations}

Before engaging in regression modeling we examined differences in Type D and illness process variables between chronic illness and healthy control groups, as well as between participants with functional somatic syndromes and illnesses of known etiology (see Table 1). When Type D was treated as a categorical variable, chi-square tests indicated that the rate of Type D personality was significantly lower in healthy controls $(39.0 \%)$ than in the illnesses of known etiology group $(52.3 \%, \mathrm{p}<.05)$ and the functional somatic syndrome group $(54.0 \%, \mathrm{p}<.05)$, but there was no significant difference between the two chronic illness groups. Group means and standard deviations along with an overall ANOVA and posthoc tests for Type D subscales and illness process variables are also presented in Table 1 . In terms of Type D subscales, negative affectivity was higher in the illnesses of known etiology and functional somatic syndrome groups than in the healthy controls, while social inhibition was only higher in the functional somatic syndrome group compared to healthy controls. 
Table 1.

Descriptive Statistics and Significance Tests of Differences between Means for Healthy, Illnesses of Known Etiology, and Functional Somatic Syndrome groups.

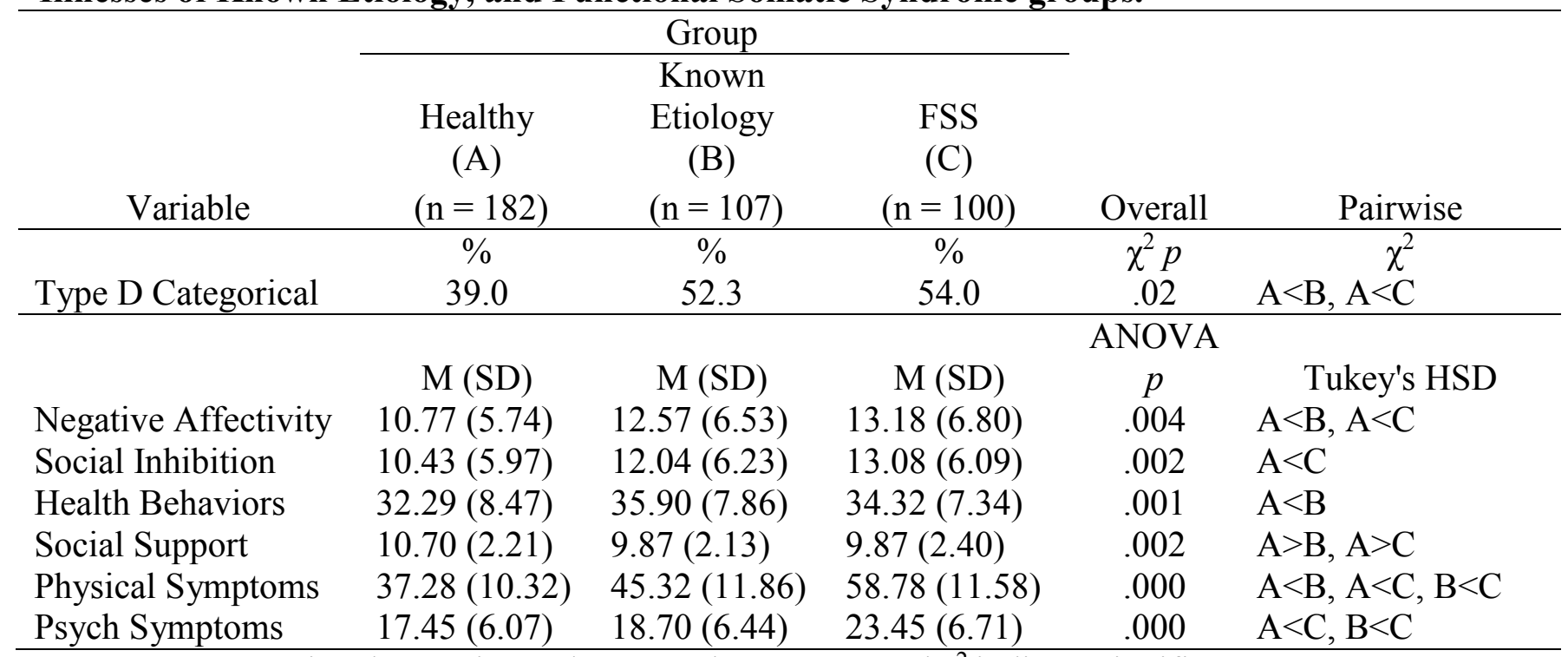

Note. FSS $=$ Functional somatic syndrome. Tukey's HSD and $\chi^{2}$ indicate significant group difference $(\mathrm{p}<.05)$.

The correlations between Type D and health-related variables for the healthy controls and the chronic illness group are presented in Table 2. Several strong correlations were present between Type D and illness process variables. The pattern of correlations was similar for both healthy and chronic illness groups with the exception that the correlation between social inhibition and health behaviors was smaller in the chronic illness group. Correlations of Type D subscales with illness process variables were generally larger for negative affectivity than for social inhibition.

Table 2.

Correlation coefficients for healthy (upper diagonal) and chronic illness participants (lower diagonal) on all variables.

\begin{tabular}{|c|c|c|c|c|c|c|}
\hline & 1 & 2 & 3 & 4 & 5 & 6 \\
\hline 1. Negative Affectivity & & .52 & -.37 & -.51 & .40 & .71 \\
\hline 2. Social Inhibition & .54 & & -.32 & -.53 & .21 & .37 \\
\hline 3. Health Behaviors & -.30 & -.07 & & .29 & -.18 & -.37 \\
\hline 4. Social Support & -.50 & -.42 & .28 & & -.28 & -.42 \\
\hline 5. Physical Symptoms & .35 & .17 & -.33 & -.29 & & .67 \\
\hline 6. Psych Symptoms & .74 & 44 & -.35 & -.46 & 65 & \\
\hline
\end{tabular}

Note. Chronic illness group $(\mathrm{n}=207)$ correlations are presented in lower diagonal; healthy control group correlations $(\mathrm{n}=182)$ are presented in upper diagonal. Significant correlations $(\mathrm{p}<.05)$ are bolded.

\subsection{Models of Type $D$ and Health Outcomes}

Linear regression was used to model the effect of Type D and illness group on health behaviors, social support, and symptom severity (physical and psychological). To facilitate 
interpretation of regression coefficients, all numeric variables in the models were Z-score standardized. Chronic illness was coded 0 for healthy controls and 1 for chronic illness. The effect of having a functional somatic syndrome was coded 0 for healthy controls or illnesses of known etiology, and 1 for functional somatic syndrome.

Before fitting regression models predicting health outcomes (i.e., health behaviors, social support, physical and psychological symptoms), we first performed a systematic comparison of the predictive validity of different representations of Type D. Specifically, for each health outcome we ran five regression models each with a different Type D representation: (1) dichotomous NA and SI main effects (i.e., based on cut-off scores of greater than or equal to 10); (2) dichotomous NA and SI main effects and interaction, which is also equivalent to including the four categories of low NA/SI, high NA only, high SI only, high NA/SI as per Denollet et al. (2013); (3) continuous NA and SI main effects; (4) continuous NA and SI main effects and interaction, (5) dichotomous Type D, (6) continuous Type D (Product), (7) continuous Type D (Sum). We obtained adjusted r-squared values for each regression (see Table 3). Results showed that dichotomous Type D was the weakest predictor (average adjusted $r$-squared $=.126$ ). Of the two continuous composite measures, the sum of NA and SI (average adjusted r-squared $=.242$ ) was better than the product (average adjusted r-squared $=.213$ ). However, reflecting the differential influence of NA and SI in predicting health outcomes, including continuous NA and SI as separate main effects provided superior prediction (average adjusted $r$-squared $=.275$ ). Adding the interaction in addition to continuous NA and SI main effects resulted in only slightly greater prediction (average adjusted $r$-squared $=.279$ ). Specifically, the NA by SI interaction only led to a significant $r$-squared change for health behavior. Dichotomous NA and SI resulted in poorer prediction than continuous NA and SI, but the general pattern of the interaction providing minimal benefit over the main effects still held. We also performed the above regressions separately for healthy and chronic illness groups and the same relative ranking of regressions emerged. Given these results, continuous representations of NA, SI, and the NA by SI interaction were included in subsequent regression models.

Table 3.

Variance Explained in Health Behaviors, Social Support, and Symptom Severity from Alternative Type D Representations using Linear Regression

\begin{tabular}{|c|c|c|c|c|c|}
\hline \multirow[b]{2}{*}{ Predictors } & $\begin{array}{c}\text { Health } \\
\text { Behavior }\end{array}$ & $\begin{array}{c}\text { Social } \\
\text { Support }\end{array}$ & $\begin{array}{l}\text { Physical } \\
\text { Symptoms }\end{array}$ & $\begin{array}{l}\text { Psych. } \\
\text { Symptoms }\end{array}$ & \multirow[b]{2}{*}{$\begin{array}{c}\text { Average } \\
\text { Adjusted } \\
\mathrm{R}^{2}\end{array}$} \\
\hline & $\begin{array}{c}\text { Adjusted } \\
\mathrm{R}^{2}\end{array}$ & $\begin{array}{l}\text { Adjusted } \\
\mathrm{R}^{2}\end{array}$ & $\begin{array}{l}\text { Adjusted } \\
\mathrm{R}^{2}\end{array}$ & $\begin{array}{l}\text { Adjusted } \\
\mathrm{R}^{2}\end{array}$ & \\
\hline $\begin{array}{l}\text { 1. Dichotomous NA and SI Main } \\
\text { effects }\end{array}$ & .059 & .234 & .088 & .348 & .182 \\
\hline $\begin{array}{l}\text { 2. Dichotomous NA and SI Main } \\
\text { effects and interaction }\end{array}$ & .068 & .233 & .086 & .347 & .183 \\
\hline $\begin{array}{l}\text { 3. Continuous NA and SI Main } \\
\text { effects }\end{array}$ & .079 & .332 & .153 & .538 & .275 \\
\hline $\begin{array}{l}\text { 4. Continuous NA and SI Main } \\
\text { effects and interaction }\end{array}$ & .092 & .332 & .154 & .538 & .279 \\
\hline 5. Dichotomous Type D & .021 & .195 & .064 & .223 & .126 \\
\hline 6. Continuous Type D (Product) & .040 & .318 & .107 & .386 & .213 \\
\hline 7. Continuous Type D (Sum) & .063 & .333 & .131 & .443 & .242 \\
\hline
\end{tabular}

Note. $\mathrm{n}=389$. Average adjusted $\mathrm{R}^{2}$ values represent average variance explain for predictor set averaged over the four illness process outcome variables. 
To examine whether the effect of Type D on health outcomes varied by clinical or functional somatic syndrome grouping variables, regression models predicting health behavior, social support, physical symptoms, and psychological symptoms were compared with and without interaction terms. Specifically, we included the six interactions created by crossing one of the Type D predictors (i.e., NA, SI, or the NA by SI interaction) with one of chronic illness indicator variables (i.e., chronic illness indicator or functional somatic syndrome indicator). Of the 24 interaction terms examined, two were statistically significant. First, the negative effect of negative affectivity on healthy behavior was reduced in the chronic illness group. Second, the negative effect of negative affectivity on social support was amplified in the functional somatic syndrome group. Thus, the hypothesis that Type D would have a differential effect by group was partially supported. As a result, subsequent regression predicting healthy behavior and social support retained group by Type D interactions. Because there no significant interactions in predicting symptom measures, interactions were excluded.

Table 4.

Regression Analysis of Type D predicting Health Behavior and Social Support

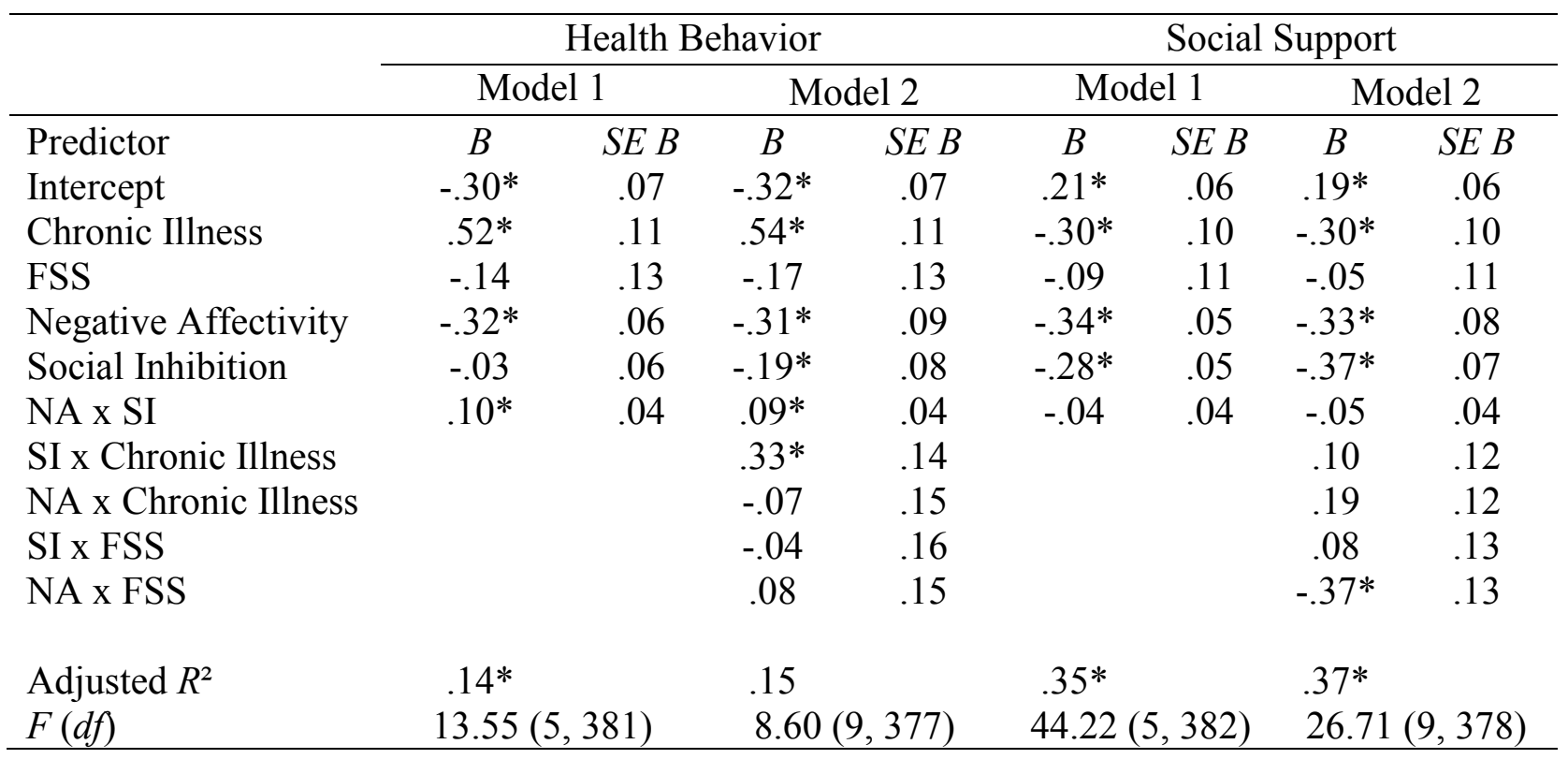

Note. Chronic illness was coded $0=$ healthy, $1=$ illnesses of known etiology or functional somatic syndrome. Functional somatic syndrome (FSS) was coded $0=$ healthy or illnesses of known etiology, 1 = FSS. Negative affect (NA), social inhibition (SI), health behavior and social support were coded as z-scores. NA x SI was the product of NA and SI z-scores. $* \mathrm{p}<.05$

To examine the effect of Type D and group membership on health behavior and social support, we fit two regression models for each outcome variable where Model 1 included illness group indicators and Type D variables, and Model 2 added negative affectivity by group and social inhibition by group interactions. Coefficients and model fits are shown in Table 4. Chronic illness participants reported more positive health behaviors and less social support, but there was no effect of having a functional somatic syndrome compared to an illnesses of known etiology. With regards to Type $\mathrm{D}$, the effect varied by outcome measure. Specifically, negative affectivity and social inhibition were equally predictive of social support, but negative affectivity was the more consistently important predictor of health 
behavior. Also, in contrast to Type D theory, the sign of the interaction suggests that the combined effect of social inhibition and negative affectivity leads to an effect less than implied by the two main effects. Nonetheless, given the small size of the effect, and that this was the only significant NA by SI interaction across the four regressions, it is appropriate to treat the result with caution. Finally, there were the two significant group by Type D interactions discussed earlier.

To examine the combined effect of Type D and the health-related mechanisms of social support and health behaviors on symptom reporting, we fit two regression models for both physical and psychological symptoms (see Table 5). Model 1 included illness group indicators and Type D variables and Model 2 added social support and health behaviors as predictors. For both physical and psychological symptoms, the functional somatic syndrome group reported many more symptoms, yet the effect of chronic illness was only significant for physical symptoms. With regards to Type D, negative affectivity but not social inhibition predicted greater levels of symptom reporting; this was particularly true for psychological symptoms. Adding health behaviors and social support to the model resulted in a small increase in variance explained with both variables predicting lower levels of both psychological and physical symptoms.

Table 5.

Regression Analysis for variables predicting Physical symptoms \& Psychological Symptoms

\begin{tabular}{|c|c|c|c|c|c|c|c|c|}
\hline \multirow[b]{3}{*}{ Predictor } & \multicolumn{4}{|c|}{ Physical Symptoms } & \multicolumn{4}{|c|}{ Psychological Symptoms } \\
\hline & \multicolumn{2}{|c|}{ Model 1} & \multicolumn{2}{|c|}{ Model 2} & \multicolumn{2}{|c|}{ Model 1} & \multicolumn{2}{|c|}{ Model 2} \\
\hline & $B$ & $S E B$ & $B$ & $S E B$ & $B$ & $S E B$ & $B$ & $S E B$ \\
\hline Intercept & $-.47 *$ & .06 & $-.48 *$ & .06 & $-.14^{*}$ & .05 & $-.15 *$ & .05 \\
\hline Chronic Illness & $.49 *$ & .09 & $.51^{*}$ & .09 & -.01 & .08 & .01 & .08 \\
\hline FSS & $.92 *$ & .10 & $.90 *$ & .10 & $.62 *$ & .09 & $.60 *$ & .09 \\
\hline Negative Affectivity & $.33^{*}$ & .04 & $.26^{*}$ & .05 & $.69 *$ & .04 & $.63 *$ & .04 \\
\hline Social Inhibition & -.03 & .04 & -.07 & .05 & .02 & .04 & -.01 & .04 \\
\hline NAxSI & -.05 & .03 & -.04 & .03 & -.03 & .03 & -.02 & .03 \\
\hline Social Support & & & $-.11 *$ & .05 & & & $-.08 *$ & .04 \\
\hline $\begin{array}{l}\text { Health } \\
\text { Behaviors }\end{array}$ & & & $-.10 *$ & .04 & & & $-.10 *$ & .03 \\
\hline Adjusted $R^{2}$ & $.47 *$ & & $.49^{*}$ & & $.61^{*}$ & & $.62 *$ & \\
\hline$F(d f)$ & 70.16 & ,381) & 53.48 & 7,379) & 120.0 & $5,381)$ & 90.72 & 379) \\
\hline
\end{tabular}

Note. Chronic illness was coded $0=$ healthy, $1=$ illnesses of known etiology or functional somatic syndrome. Functional somatic syndrome (FSS) was coded $0=$ healthy or illnesses of known etiology, 1 = FSS. Negative affect (NA), social inhibition (SI), health behavior, social support, physical symptoms, and psychological symptoms were coded as z-scores. NA x SI was the product of NA and SI z-scores.

$* \mathrm{p}<.05$

\section{Discussion}

The present study aimed to extend the literature on Type D personality by investigating the presence and effect of Type D in a range of chronic illness groups. First, Type D prevalence was greater in chronic illness participants than in healthy controls, 
however, contrary to our prediction, there was no significant difference in the rate of Type D between functional somatic syndromes and illnesses of known etiology. Second, models of the effect of Type D on health behaviors, social support, and symptom severity indicated that social inhibition and negative affectivity had differential effects with negative affectivity generally having the stronger impact, except in the case of social support where the effects were of a similar magnitude. Third, building on the previous point, including social support and negative affect as separate predictors led to much better prediction of health outcomes and symptom reporting than using only categorical Type $\mathrm{D}$ or any continuous sum or product composite of Type D. Fourth, while Type D predicted health behaviors, social support, and physical symptoms, the effect of Type D on symptoms appeared to be more direct, as opposed to operating through these potential mediators. Fifth, there were a small number of interactions between chronic illness group and Type D in predicting health behaviors, social support, and symptom severity. Overall, the results support the suggestion that Type D may be a significant factor in chronic illnesses beyond those associated with cardiac health.

\subsection{Type D Prevalence in Chronic Illness}

The prevalence of Type D was higher in the chronic illness group (53.6\%) than in healthy controls $(39.2 \%)$. The rate of Type D in the healthy controls was similar to other Australian population estimates $(39.7 \%$, Horwood et al., 2014) but higher than reported international estimates (24\%, Mols \& Denollet, 2010a). The rate in the chronic illness group was similar to hypertensive cardiac patients, who, in turn, had the highest rate of all cardiac patients (53\%, Pedersen \& Denollet, 2006). A similar pattern emerged when looking at Type D subscales with both social inhibition and negative affectivity being generally higher in chronic illness groups, although the data suggested that differences with healthy controls were greater for functional somatic syndromes than illnesses of known etiology. It may be that the continuous measures of Type D provided a more nuanced estimate of the differences between groups than is provided by categorical prevalence estimates.

There are several possible explanations for the observed differences. First, it may be that merely having a chronic illness is sufficient to make people experience more negative emotions and reduce engagement in social interactions. Second, pre-morbid Type D individuals are likely to engage in fewer positive health behaviors than pre-morbid non-Type D's; thus Type D may contribute to acquiring a chronic illness. Third, the trend in the data suggesting higher levels of negative affect and social inhibition in people with a functional somatic syndrome amplifies, or may reflect, the psychological mechanisms of the conditions. Finally, the differences also add weight to the proposal that Type D may have an indirect effect on symptoms via health behaviors and social support pathways.

\subsection{Type $D$ and Health Outcomes}

Type D theory implies that a categorical representation is better than a continuous representation and that negative affectivity and social inhibition have a multiplicative effect. In response to the loss of prediction that occurs when converting a continuous variable into a dichotomous one, researchers have tried using both sums (Horwood et al., 2015) and products (Kelly-Hughes et al., 2014; Stevenson \& Williams, 2014) of Type D subscales to create continuous scores for Type D. Consistent with more parsimonious principles of personality trait theory, results from the representational analysis challenge the categorical and multiplicative representations of Type D. Continuous Type D predicted better than categorical, the sum of negative affectivity and social inhibition predicted better than the product, and treating negative affectivity and social inhibition as separate predictors allowed for better prediction of health outcomes than either composite of negative affectivity and social inhibition. Furthermore, with the exception of health behaviors, the continuous forms of Type D did not provide incremental prediction, and even in the case of health behaviors, 
the interaction effect was in the opposite direction to theory. These results are broadly consistent with findings from Stevenson and Williams (2014) and Kelly-Hughes et al (2014) showing that the interaction between negative affectivity and social inhibition rarely adds significant prediction over and above main effect. Rather, a better interpretation is that the two subscales are important predictors that operate as separate main effects. Importantly, negative affectivity appears to be a stronger predictor of health outcomes than social inhibition, although notable exceptions exist where the health related variable has a strong social component. Given the differential role of Type D predictors on health outcomes (e.g., social inhibition on social support), a Type D composite may hide these differential effects.

Another broad question was whether Type D had a differential effect on health outcomes between healthy and chronic illness groups and between functional somatic syndromes and illnesses of known etiology. Given the overlap of mechanisms associated with both Type D and functional somatic syndrome such as poor health behaviors, low perception of social support, adoption of poor coping mechanisms and greater reporting of somatic complaints (Mols \& Denollet, 2010b), we expected to find such interactions. While results indicated two significant interactions at the .05 level, we treat the results cautiously given that they were not significant at the Bonferroni adjusted .002 level. These two interactions showed that the effect of negative affect on social support was amplified in the functional somatic syndrome group and that the effect of social inhibition on health behaviors was reduced in the chronic illness group. Thus, on balance, there is more evidence that the relationship between Type D personality and health outcomes is similar across illnesses. This suggests that models of Type D personality may generalize across different illnesses.

A theme explored in the results is the extent to which symptoms could be explained by Type D personality versus process variables such as health behaviors and social support. The theory of Type D suggests that Type D leads to a general inability to cope with stress and seek help, which can, in turn, lead to avoidance behaviors followed by health problems. In contrast, while Type D is associated with process variables that were related to symptoms, there was also support for a more direct role of negative affectivity. This was particularly evident when looking at the relationship between negative affectivity and psychological symptoms, where a very strong relationship was observed. This is broadly consistent with negative affectivity providing a general negative lens through which people experience both clinical and non-clinical health issues. It also made sense that this negative lens would be more relevant to psychological symptoms, which are arguably less constrained by the external world than physical symptoms. These results are also consistent with previous research that found Type $\mathrm{D}$ to be associated with higher rates of musculoskeletal pain and psychological symptom reporting (Condén et al., 2013). It may be that as the rate of negative affectivity and social inhibition increases, so does the subjective experience of illness and illness-related symptoms. Future research should examine whether the subjective experience of symptoms is consistent with objective measures of illness severity.

Finally, prior research has presented evidence of a relationship between Type D, health behaviors, social support and symptom reporting (Svansdottir, van den Broek, Karlsson, Gudnason, \& Denollet, 2012; Williams et al., 2008) in healthy and cardiac populations. The results of the present study offer some support to the proposed relationship, however it does appear that negative affectivity is the primary predictor in most cases. In predicting symptoms, health behavior and social support had incremental prediction, suggesting that there may be a cumulative effect of Type D with health behaviors and social support on reported symptom severity.

\subsection{Limitations and Future Research}

Several limitations in the present study are worth noting. First, the sample obtained had a large proportion of females. Females are more likely to report somatic symptoms than 
males (APA, 2013), however Type D personality appears to occur relatively evenly between males and females (Mols et al., 2012). Second, the cross-sectional nature of the research means that causal relationships between Type D, illness, and health outcomes could not be answered. Third, the study had a limited ability to investigate Type D within specific illness groups. The data were collapsed across illnesses and across functional somatic syndrome status in order to ensure adequate power was achieved in the analyses. Further research could aim to develop a larger sample in order to look for more subtle effects of Type D and illness type. Finally, the data were obtained via self-report questionnaire, hence we are unable to ascertain the degree to which participant's perceptions of social support or symptom severity are consistent with objective measures.

\subsection{Conclusion}

Overall, the current study contributes to a number of aspects of Type D research. While Type D may be a useful diagnostic heuristic for clinicians, predictive models clearly favor treating the subscales of Type $\mathrm{D}$ as continuous additive effects; the greater importance of negative affectivity and the absence of interaction effects between Type D subscales provides a further challenge to the novelty of the Type D construct. More broadly, the present study expanded Type D research to previously untested chronic illnesses, finding that models of Type D developed in CVD patients appear to be more generally applicable. These findings not only help to better understand the construct, but may assist in developing better models of personality and health outcomes for use in clinical and applied health-care settings. Specific reference to personality variables is often absent in health determinant models, however increasing evidence from Type D research suggests that particular traits, such as those captured by measures of Type $\mathrm{D}$, represent important risk factors for health behaviors, illness perceptions and overall health status. 


\section{References}

APA. (2013). Diagnostic and statistical manual of mental disorders (5th ed.). Washington, DC.

Bergvik, S., Sørlie, T., Wynn, R., \& Sexton, H. (2010). Psychometric properties of the Type D scale (DS14) in Norwegian cardiac patients. Scandinavian Journal of Psychology, 51(4), 334-340. doi: 10.1111/j.14679450.2009.00793.x

Chan, J. K. Y., \& Consedine, N. S. (2014). Negative affectivity, emotion regulation, and coping in migraine and probable migraine: a new zealand case-control study. International Journal Of Behavioral Medicine, 21(5), 851-860. doi: 10.1007/s12529-013-9370-6

Condén, E., Leppert, J., Ekselius, L., \& Åslund, C. (2013). Type D personality is a risk factor for psychosomatic symptoms and musculoskeletal pain among adolescents: a cross-sectional study of a large populationbased cohort of swedish adolescents. BMC Pediatrics, 13(11)

Condén, E., Rosenblad, A., Ekselius, L., \& Aslund, C. (2014). Prevalence of Type D personality and factorial and temporal stability of the DS14 after myocardial infarction in a Swedish population. Scandinavian Journal Of Psychology, 55, 601-610.

Conraads, V. M., Denollet, J., De Clerck, L. S., Stevens, W. J., Bridts, C., \& Vrints, C. J. (2006). Type D personality is associated with increased levels of tumour necrosis factor (TNF)-alpha and TNF-alpha receptors in chronic heart failure. Int J Cardiol, 113(1), 34-38. doi: 10.1016/j.ijcard.2005.10.013

Dalgard, O. S., Bjørk, S., \& Tambs, K. (1995). Social support, negative life events and mental health. The British Journal of Psychiatry, 166, 29-34. doi: 10.1192/bjp.166.1.29

De Haes, J., \& Van Knippenberg, F. (1990). Rotterdam Symptom Checklist. Oncology, 4(5), 44-45.

Denollet, J. (2005). DS14: standard assessment of negative affectivity, social inhibition, and Type D personality. Psychosomatic Medicine, 67(1), 89-97. doi: 10.1097/01.psy.0000149256.81953.49

Denollet, J., \& Pedersen, S. (2008). Prognostic value of Type D personality compared with depressive symptoms. Archives Of Internal Medicine, 168(4), 431-432. doi: 10.1001/archinternmed.2007.120

Denollet, J., Pedersen, S., Ong, A. T. L., Erdman, R. A. M., Serruys, P. W., \& van Domburg, R. T. (2006). Social inhibition modulates the effect of negative emotions on cardiac prognosis following percutaneous coronary intervention in the drug-eluting stent era. European Heart Journal, 27(2), 171177.

Denollet, J., Pedersen, S. S., Vrints, C. J., \& Conraads, V. M. (2013). Predictive value of social inhibition and negative affectivity for cardiovascular events and mortality in patients with coronary artery disease: the type d personality construct. Psychosomatic Medicine, 75(9), 873-881. doi: 10.1097/PSY.0000000000000001

Denollet, J., Sys, S. U., Stroobant, N., Rombouts, H., Gillebert, T. C., \& Brutsaert, D. L. (1996). Personality as independent predictor of long-term mortality in patients with coronary heart disease. Lancet (British edition), 347(8999), 417-421.

Dubayova, T., Krokavcova, M., Nagyova, I., Rosenberger, J., Gdovinova, Z., Middel, B., Groothoff, J. W., \& van Dijk, J. P. (2013). Type D, anxiety and depression in association with quality of life in patients with Parkinson's disease and patients with multiple sclerosis. Quality Of Life Research: An International Journal Of Quality Of Life Aspects Of Treatment, Care And Rehabilitation, 22(6), 1353 1360. doi: 10.1007/s11136-012-0257-9

Ferguson, E., Williams, L., O’Connor, R. C., Howard, S., Hughes, B. M., Johnston, D. W., Allan, J. L., O’Connor, D. B., Lewis, C. A., \& Grealy, M. A. (2009). A taxometric analysis of type-D personality. Psychosomatic Medicine, 71(9), 981-986.

Habra, M., Linden, W., Anderson, J., Weinberg, J. (2003). Type D personality is related to cardiovascular and neuroendocrine reactivity to acute stress. Journal of Psychosomatic Research, 55, 235- 245. doi: 10.1016/S0022-3999(02)00553-6

Haslam, N., Holland, E., \& Kuppens, P. (2012). Categories versus dimensions in personality and psychopathology: a quantitative review of taxometric research. Psychological medicine, 42(05), 903920. doi: $10.1017 / \mathrm{S} 0033291711001966$

Horwood, S., Anglim, J., \& Tooley, G. (2015). Type D personality and the Five-Factor Model: A facet-level analysis. Personality and Individual Differences, 83, 50-54.

Horwood, S., Chamravi, D., \& Tooley, G. (2014). Examining the prevalence of Type-D personality in an Australian population. Australian Psychologist, 50, 212-218. doi: 10.1111/ap.12089

Husson, O., Denollet, J., Oerlemans, S., \& Mols, F. (2013). Satisfaction with information provision in cancer patients and the moderating effect of Type D personality. Psycho-Oncology, 22(9), 2124-2132. doi: $10.1002 /$ pon. 3267

Jellesma, F. C. (2008). Health in young people: social inhibition and negative affect and their relationship with self-reported somatic complaints. Journal of Developmental \& Behavioral Pediatrics, 29(2), 94-100.

Kelly-Hughes, D. H., Wetherell, M. A., \& Smith, M. A. (2014). Type D personality and cardiovascular reactivity to an ecologically valid multitasking stressor. Psychology and Health, 29(10), 1156-1175. 
Kupper, N., Gidron, Y., Winter, J., \& Denollet, J. (2009). Association between type D personality, depression, and oxidative stress in patients with chronic heart failure. Psychosomatic Medicine, 71(9), 973-980.

Martin, L., Doster, J., Critelli, J., Purdum, M., Powers, C., Lambert, P., \& Miranda, V. (2011). The 'distressed' personality, coping and cardiovascular risk. Stress \& Health: Journal of the International Society for the Investigation of Stress, 27(1), 64-72.

Michal, M., Wiltink, J., Grande, G., Beutel, M., \& Brähler, E. (2011). Type D personality is independently associated with major psychosocial stressors and increased health care utilization in the general population. Journal Of Affective Disorders, 134(1-3), 396-403. doi: http://dx.doi.org/10.1016/j.jad.2011.05.033

Molloy, G. J., Perkins-Porras, L., Strike, P. C., \& Steptoe, A. (2008). Type-D Personality and Cortisol in Survivors of Acute Coronary Syndrome. Psychosomatic Medicine, 70(8), 863-868. doi: 10.1097/PSY.0b013e3181842e0c

Mols, F., \& Denollet, J. (2010a). Type D personality among noncardiovascular patient populations: a systematic review. General hospital psychiatry, 32(1), 66-72.

Mols, F., \& Denollet, J. (2010b). Type D personality in the general population: a systematic review of health status, mechanisms of disease, and work-related problems. Health \& Quality of Life Outcomes, 8, 110. doi: 10.1186/1477-7525-8-9

Mols, F., Denollet, J., Kaptein, A. A., Reemst, P. H. M., \& Thong, M. S. Y. (2012). The association between Type D personality and illness perceptions in colorectal cancer survivors: A study from the populationbased PROFILES registry. Journal of Psychosomatic Research, 73, 232-239. doi: 10.1016/j.jpsychores.2012.07.004

Mommersteeg, P. M. C., Kupper, N., \& Denollet, J. (2010). Type D personality is associated with increased metabolic syndrome prevalence and an unhealthy lifestyle in a cross-sectional Dutch community sample. BMC Public Health, 10, 714-714.

Nefs, G., Speight, J., Pouwer, F., Pop, V., Bot, M., \& Denollet, J. (2015). Type D personality, suboptimal health behaviors and emotional distress in adults with diabetes: Results from Diabetes MILES-The Netherlands. Diabetes Research \& Clinical Practice, 108(1), 94-105. doi: 10.1016/j.diabres.2015.01.015

Pedersen, S., \& Denollet, J. (2004). Validity of the Type D personality construct in Danish post-MI patients and healthy controls. Journal of Psychosomatic Research, 57, 265-272. doi: 10.1016/S00223999(03)00614-7

Pedersen, S., \& Denollet, J. (2006). Is Type D Personality Here to Stay? Emerging Evidence Across Cardiovascular Disease Patient Groups. Current Cardiology Reviews, 2(3), 205-213. doi: 10.2174/157340306778019441

Pedersen, S., \& Schiffer, A. A. (2011). The distressed (Type D) personality. A risk marker for poor health outcomes in ICD patients. Herzschrittmachertherapie \& Elektrophysiologie, 22(3), 181-188. doi: 10.1007/s00399-011-0139-9

Pelayo-Alvarez, M., Perez-Hoyos, S., \& Agra-Varela, Y. (2013). Reliability and concurrent validity of the Palliative Outcome Scale, the Rotterdam Symptom Checklist, and the Brief Pain Inventory. J Palliat Med, 16(8), 867-874. doi: 10.1089/jpm.2012.0625

Pelle, A., Schiffer, A., Smith, O., Widdershoven, J., \& Denollet, J. (2010). Inadequate consultation behavior modulates the relationship between Type D personality and impaired health status in chronic heart failure. International Journal of Cardiology, 142, 65-71. doi: 10.1016/j.ijcard.2008.12.086

Polman, R., Borkoles, E., \& Nicholls, A. R. (2010). Type D personality, stress, and symptoms of burnout: The influence of avoidance coping and social support. British Journal of Health Psychology, 15(3), 681696.

Romppel, M., Herrmann-Lingen, C., Vesper, J., \& Grande, G. (2012). Six year stability of Type-D personality in a German cohort of cardiac patients. Journal Of Psychosomatic Research, 72(2), 136-141.

Rosmond, R., \& Björntorp, P. (2000). The hypothalamic-pituitary-adrenal axis activity as a predictor of cardiovascular disease, type 2 diabetes and stroke. Journal of Internal Medicine, 247(2), 188-197. doi: 10.1046/j.1365-2796.2000.00603.x

Sajadinejad, M. S., Molavi, H., Asgari, K., Kalantari, M., \& Adibi, P. (2012). Personality dimensions and type D personality in female patients with ulcerative colitis. Journal Of Research In Medical Sciences: The Official Journal Of Isfahan University Of Medical Sciences, 17(10), 898-904.

Stevenson, C., \& Williams, L. (2014). Type D personality, quality of life and physical symptoms in the general population: a dimensional analysis. Psychology \& Health, 29(3), 365-373.

Svansdottir, E., van den Broek, K. C., Karlsson, H. D., Gudnason, T., \& Denollet, J. (2012). Type D personality is associated with impaired psychological status and unhealthy lifestyle in Icelandic cardiac patients: a cross-sectional study. BMC Public Health, 12, 42-42. 
Vollrath, M., \& Torgersen, S. (2002). Who takes health risks? A probe into eight personality types. Personality and Individual Differences, 32, 1185-1197. doi: 10.1016/S0191-8869(01)00080-0

Watson, D., \& Pennebaker, J. W. (1989). Health complaints, stress, and distress: exploring the central role of negative affectivity. Psychological review, 96(2), 234-254.

Whitehead, D. L., Perkins-Porras, L., Strike, P. C., Magid, K., \& Steptoe, A. (2007). Cortisol awakening response is elevated in acute coronary syndrome patients with type-D personality. Journal of Psychosomatic Research, 62, 419-425. doi: 10.1016/j.jpsychores.2006.11.005

Williams, L., O'Connor, R. C., Howard, S., Hughes, B. M., Johnston, D. W., Hay, J. L., O'Connor, D. B., Lewis, C. A., Ferguson, E., Sheehy, N., Grealy, M. A., \& O'Carroll, R. E. (2008). Type-D personality mechanisms of effect: The role of health-related behavior and social support. Journal of Psychosomatic Research, 64(1), 63-69. doi: http://dx.doi.org/10.1016/j.jpsychores.2007.06.008

Wongpakaran, T., \& Wongpakaran, N. (2014). Personality traits influencing somatization symptoms and social inhibition in the elderly. Clinical interventions in aging, 9, 157.

Zohar, A. H., Denollet, J., Lev Ari, L., \& Cloninger, C. (2011). The psychometric properties of the DS14 in Hebrew and the prevalence of Type D personality in Israeli adults. European Journal of Psychological Assessment, 27(4), 274-281. doi: 10.1027/1015-5759/a000074 